\title{
VULNERABILIDAD DEL COMERCIO EXTERIOR PERUANO EN EL CONTEXTO GLOBAL ACTUAL
}

\author{
PERUVIAN INTERNATIONAL TRADE VULNERABILITY IN THE \\ CURRENT GLOBAL CONTEXT \\ Francisca Bouby Tolentino* \\ Docente Principal de la Facultad de Ciencias Administrativas \\ Universidad Nacional Mayor de San Marcos - UNMSM / Lima - Perú \\ [Recepción: Agosto 2016/ Conformidad: Setiembre 2016]
}

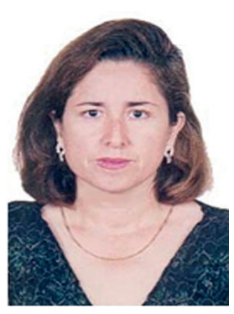

\section{RESUMEN}

El proceso de liberalización de la economía peruana iniciado en los años noventa, ha conducido al sector externo a un alto nivel de vulnerabilidad en el contexto global actual. Por un lado, el acelerado crecimiento de las exportaciones, fundamentalmente, de productos primarios, está ligado al incremento de precios por la fuerte demanda China de estos productos, a partir de la década del 2000; sin embargo, la crisis internacional de 2008-2009 y el inicio del cambio de modelo económico chino, incidieron en el proceso de declive de las exportaciones peruanas a partir del año 2012; acentuado por la fuerte caída de los precios de las materias primas y la pérdida de competitividad frente a los productos asiáticos. De otro lado, la estructura y composición del comercio exterior peruano, con un patrón de especialización orientado a la exportación de bienes de poco valor agregado y la importación creciente de bienes de alta y mediana tecnología, representa una de las mayores debilidades de la economía nacional.

\section{Palabras Clave:}

Liberalización comercial; crecimiento económico, competitividad; crisis.

\begin{abstract}
The liberalization process of the Peruvian economy, initiated in the 1990s, has led the external sector to a high level of vulnerability in the current global context. On the one hand, the rapid growth of exports -mainly primary products- is linked to the price increase due to China's strong demand, starting in the 2000s. However, the international crisis of 2008-2009 and the beginning of the Chinese economic model change, had an impact on the process decline of Peruvian exports since 2012; accentuated by the sharp fall in raw material prices and the loss of competitiveness vis-à-vis Asian products. On the other hand, the structure and composition of Peruvian international trade, including a pattern of specialization oriented to the export of low added value goods and the growing import of high and medium technology goods, represent one of the major weaknesses of the national economy.
\end{abstract}

\section{Keywords:}

Commercial liberalization, Economic growth, competitiveness, crisis.

\footnotetext{
*Doctora en Economía (UNAM, México, D.F.), Magíster en Economía -UNMSM, Economista -UNMSM. E.mail: f_bouby@yahoo.com
} 


\section{INTRODUCCIÓN}

El comercio exterior tiene que ver con los momentos históricos, culturales y económicos que vive el país en un contexto internacional cambiante con crisis recurrentes (Contreras, 2002). El contexto global que enmarca el proceso de liberalización de las economías en desarrollo como la economía peruana, a partir de los años noventa, se desarrolla como un proceso de auge y crisis del comercio internacional (OMC, 2014; CEPAL, 2015). En el contexto latinoamericano, destaca China como socio estratégico en el destino de sus exportaciones, cuya desaceleración en el crecimiento de su economía es motivo de preocupación, principalmente para la economía peruana (Perroti, 2015). El proceso de liberalización comercial de la economía peruana, implementado desde los años noventa, ha generado alta vulnerabilidad del comercio exterior, en el contexto global actual, acentuado por la composición y estructura del comercio altamente dependiente de las exportaciones de productos primarios (Roca, 2012; Adex, 2015).

El presente estudio de investigación tiene por objetivo analizar, desde un punto de vista crítico, el nivel de vulnerabilidad del comercio exterior peruano, como resultado del proceso de liberalización de la economía, en un contexto global de crisis recurrentes.

Justificación: El Comercio exterior, desde mediados del año 2011, presenta una contracción de las exportaciones, debido a la desaceleración de la economía, por la crisis de los bonos soberanos europeos que representó un problema más grande que la crisis hipotecaria de Estados Unidos. Hacia 2012, nuestras exportaciones tradicionales habían caído -3.3\% y las no tradicionales habían reducido su ritmo de crecimiento a la tercera parte de los años anteriores. (Adex, 2015). El bienio 2013-2014 el sector exportador ve minada su capacidad competitiva; es así que las exportaciones como porcentaje del PBI ha regresado a la participación que tenía hacia 1978. El enfoque teórico empleado en el análisis del comercio exterior, parte desde un punto de vista crítico al modelo neoliberal implementado en la economía actual.

\section{MARCO TEÓRICO}

Los sectores económicos y políticos dominantes, vienen realizando grandes esfuerzos para convencer a la sociedad, que los postulados de la libre competencia bases del libre mercado, la apertura comercial y los tratados comerciales, son los instrumentos para lograr un mayor crecimiento económico y elevación de la calidad de vida.

Los tratados comerciales que se vienen generando se sostienen en la teoría neoliberal de la integración económica, la cual está basada en los conceptos y principios de la teoría del comercio internacional.

\section{Teoría del comercio Internacional}

El supuesto implícito de la teoría tradicional del comercio internacional es la competencia perfecta. La nueva teoría del comercio internacional, elimina este supuesto y sustenta que el comercio no se debe a las diferencias de los países sino a la especialización arbitraria por el uso de economías de escala, destacadas por Smith y Ohlin. La nueva teoría, no rechaza las ventajas comparativas, sino que las complementa (Krugman, 1988: 41-46). Posteriormente, se diferencian las ventajas comparativas naturales o estáticas de las ventajas comparativas dinámicas; las primeras existen independientemente de la acción del hombre, como la ubicación geográfica de un país o la disponibilidad de recursos naturales; las segundas requieren de esfuerzo para que esa ubicación geográfica estratégica o ese recurso natural sean aprovechados cabalmente en el comercio internacional (Cornejo, 1996: 43).

Michael Porter (1991), en su obra La Ventaja Competitiva de las Naciones, rescata los aportes tanto de David Ricardo como de Schumpeter, para luego conjuntamente con Dahlman presentar su propuesta sobre la productividad y competitividad, tanto a nivel empresarial como de las naciones. David Ricardo (siglo XIX), introduce la noción de productividad al definir las ventajas comparativas que tenía un país al producir un bien, respecto a otro país que produce el mismo bien. Schumpeter (1943), argumenta que las revoluciones tecnológicas propician la transformación de las ventajas comparativas estáticas en dinámicas y en permanente flujo. Por lo tanto, 
para Porter la productividad del capitaly del trabajo, son las que hacen que una nación sea competitiva. (Bouby, 1999: 13-21)

\section{Teoría de la integración económica}

La teoría de la integración económica, está relacionada con la teoría del comercio internacional, por ello hay que distinguir entre la teoría tradicional y la nueva teoría, tanto del comercio como de la integración económica. La teoría tradicional analiza los efectos de la integración económica bajo la óptica de la estática comparativa, según los principios ricardianos de la teoría de las ventajas comparativas; la nueva teoría analiza las consecuencias dinámicas, incorporando en el análisis la competencia monopolística y las economías de escala y de alcance. Lo cual contrasta con la teoría tradicional de Viner, que descansa en la hipótesis del que el comercio mundial se realiza en un mercado de competencia perfecta, haciendo hincapié en el comercio como factor de bienestar. (Bouby, 1999: 22-25).

\section{Integración y globalización}

Existen sendos trabajos teórico-académicos que demuestran que la integración y la globalización, tal como funcionan, vienen generando una brecha social cada vez más amplia (Pickety, 2014; Krugman, 2009; Stiglitz, 2012), como resultado de la elevación de los índices de riqueza económica de unos pocos en contraposición con la disminución de la calidad de vida de las clases medias y de los sectores más desfavorecidos que irremediablemente ingresan al campo de la pobreza. Lo cual deviene en antagonismo social que no permitirá lograr la paz social que requiere el sistema capitalista para mantener el status quo.

\section{METODOLOGÍA}

Se ha empleado el método analítico-sintético. El estudio es de tipo descriptivo, correlacional, a través de una investigación documental desde una perspectiva crítica. Las fuentes de información son básicamente secundarias.

\section{RESULTADOS \\ Contexto Global: auge y crisis del comercio internacional}

La antesala de la globalización fue el neoliberalismo que comenzó a desarrollarse desde inicios de la década de los ochenta del siglo veinte. Los países capitalistas, como Inglaterra y Estados Unidos, se hallaban en recesión y venían sufriendo las consecuencias de la crisis petrolera generada por el proceso de nacionalización de los yacimientos petroleros fundamentalmente árabes. Lo cual motivó la búsqueda de recursos económicos mediante el cobro de la deuda externa en los países en desarrollo, principalmente a los latinoamericanos que se hallaban sumamente endeudados por aquel entonces, vía organismos financieros internacionales tales como el Fondo Monetario Internacional (FMI), Banco Mundial y Club de París. (Contreras, 2002: 155-157).

En esas condiciones histórico-concretas, los gobiernos latinoamericanos ante la imposibilidad del pago, se vieron obligados a implementar los Programas de Ajuste Estructural, a fin de generar los recursos necesarios para el pago de la deuda externa, lo cual devino en la venta de activos del Estado, recorte de los programas sociales, disminución de los niveles de calidad de salud y educación pública, readecuación de los regímenes de pensiones, etc. En concreto, lo que se dio a partir de los ochenta fue una apertura unilateral en las economías de América Latina.

En realidad, a partir del decenio de los ochenta, los países en desarrollo en su conjunto siguieron una tendencia aperturista, acompañada de una reducción de los aranceles que aplican en régimen de la nación más favorecida (NMF) a las importaciones (OMC, 2014: 6). Otra de las tendencias es la expansión de las cadenas de valor mundiales, según la OMC, casi la mitad de las exportaciones brutas del mundo se relacionan con cadenas de valor mundiales, donde las economías que tuvieron una mayor participación durante 1995-2008 fueron China, India, Corea, Filipinas y el Taipei chino. (OMC, 2014: 7)

La inversión extranjera directa juega un rol fun- 
damental en el establecimiento de vínculos en las cadenas de valor globales, su evolución en los países en desarrollo pone de manifiesto su participación creciente en dichas cadenas. Así en el año 2012 estos países absorbieron casi la mitad de la Inversión Extranjera Directa (IED) mundial; y también como fuente de IED, estos países representaron en conjunto el $34 \%$ en 2012 , comparado con el $7 \%$ de finales de los ochenta. (OMC, 2014: 7)

Sin embargo, los países en desarrollo enfrentan una serie de limitaciones e impedimentos para su participación en las cadenas de valor, tales como, los costos y retrasos de transporte y los procedimientos aduaneros; los derechos de importación y las prescripciones en materia de licencias; las limitaciones de acceso a la financiación del comercio y el cumplimiento de las normas.

El aumento de los precios de los productos básicos, y la importancia creciente de las exportaciones de esos productos, son características del comercio mundial. Así, entre 2003 y 2008, los precios de la energía, metales y minerales se duplicaron con creces, como consecuencia de la expansión de varias economías en desarrollo del Grupo G-20 basada en la utilización intensiva de recursos naturales y energéticos. Aunque hayan disminuido recientemente los precios de estos productos, siguen siendo elevados respecto a una década atrás. (OMC, 2014: 8).
Según la OMC, en el caso de América Latina, el aumento de los precios mundiales de los productos básicos y el consiguiente aumento de su producción y exportación habrían representado tal vez entre la tercera parte y la mitad del crecimiento de la región durante la década del 2000. (OMC, 2014: 13).

Otra tendencia de la economía actual, es el alcance cada vez más global de las crisis macroeconómicas. Así la crisis 2008-2009, mostró hasta qué punto las economías en desarrollo y las economías emergentes dependen del proceso cíclico originado en las economías desarrolladas y de los vínculos comerciales y financieros y la importancia de las cadenas de suministro en la propagación de las crisis y el agotamiento de la financiación del comercio.

Según la Comisión Económica para América Latina (CEPAL), después de la crisis global de 20082009 el comercio mundial ha perdido su dinamismo, luego de haber caído un $12 \%$ en 2009, las exportaciones (medidas en volumen) sólo crecieron un $2.6 \%$ entre 2012 y 2014, tal como se observa en la Figura $\mathrm{N}^{\circ} 01$. El escaso dinamismo del comercio mundial a partir del año 2012 se evidencia con las variaciones negativas registradas en el índice de precios de las exportaciones mundiales, en un contexto de menores precios de los productos básicos como el petróleo y los metales. (Cepal, 2015:24)

Figura No 01

MUNDO: VARIACIÓN ANUAL DE LAS EXPORTACIONES DE BIENES SEGÚN VALOR, PRCIO Y VOLUMEN 1992 - 2015

(En porcentajes)

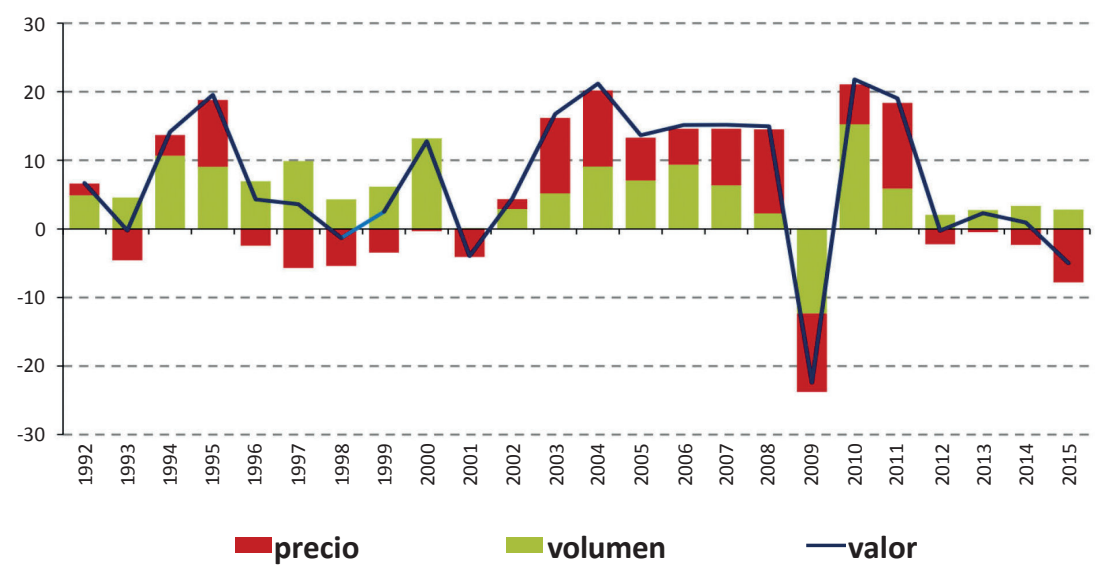

Fuente: Comisión Económica para América Latina y el Caribe (CEPAL), sobre la base de información del Netherlands Bureau for Economic Policy Analysis (CPB).

World Trade Database [en linea] http://www.cpb.nl/en/world-trade-monitor 


\section{Contexto latinoamericano:}

\section{China socio estratégico}

Durante la última década, han proliferado los vínculos entre América Latina y Asia Oriental; de cuyos países hay mucho que América Latina puede aprender en términos de estrategias de crecimiento, basadas en las exportaciones, tales como, la basta inversión pública en infraestructura que deriva en bajos costos logísticos y elevado nivel de eficiencia, lo cual alienta la inversión local e internacional, al mismo tiempo que genera una mayor competitividad. Otra lección por aprender tiene que ver con el aumento de la productividad de los factores de producción, como efecto de la inversión de capital, las innovaciones tecnológicas y la capacitación de la fuerza laboral. Sin embargo, habría que precisar que el crecimiento no podría ser sostenible si se ignoran temas como la desigualdad en el ingreso, corrupción, transparencia, normas claras y un estado de derecho.

En el área comercial, China representa un socio estratégico para América Latina. En la Figura $\mathrm{N}^{\circ} 02$ se observa que América Latina presentó un crecimiento promedio anual de sus exportaciones a China del 19\%, durante el período 1990-2013. Sin embargo, esta tasa asciende a $24 \%$ durante la primera década del presente siglo.

Figura No 02

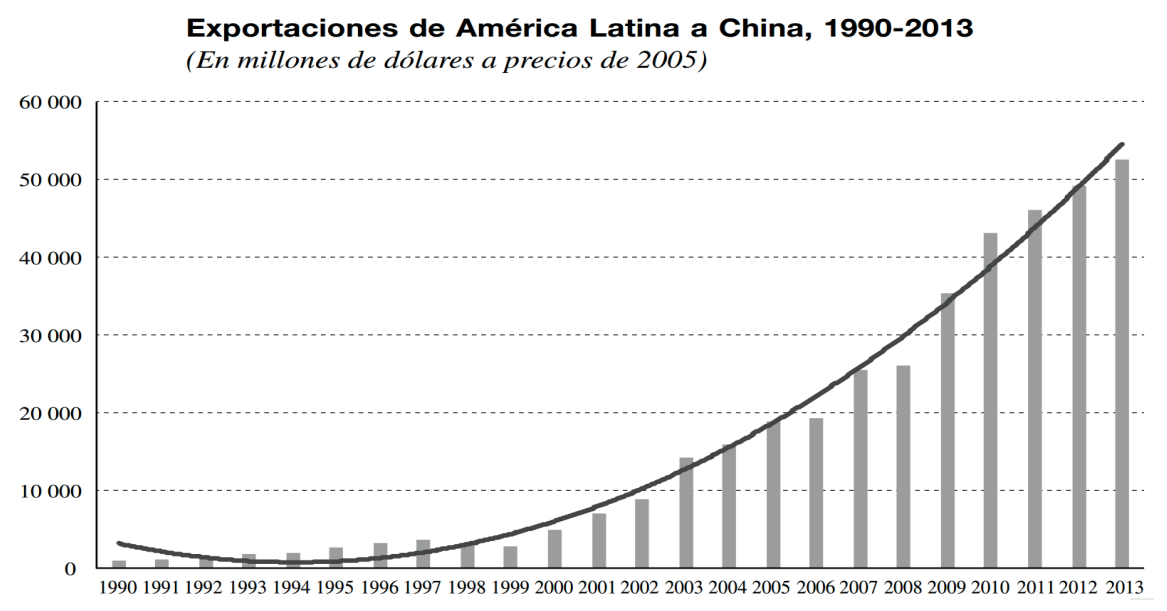

Fuente: Perroti, 2015, p. 53

El intercambio comercial se caracteriza por exportaciones de productos primarios y minerales, tales como, soja, metales y petróleo de parte de América Latina, en tanto que las importaciones concentradas en bienes manufacturados provienen de Asia, estableciéndose un patrón de intercambio tipo nuevo centro-periferia, donde claramente el nuevo centro es China. (Rosales y Kuayama, 2012, citado en Perroti, 2015).

La demanda de bienes primarios de parte de China obedeció a su proceso de industrialización, lo cual condujo a un incremento de precios, lo que significó una sustancial mejora en los términos de intercambio para varios países latinoamericanos.

El proceso de industrialización en China al pasar de bienes intensivos en trabajo, como la confección, a sectores más intensivos en capital, como los productos eléctricos y electrónicos, impulsaron fuertemente la demanda de metales, asimismo el sector construcción y los proyectos de infraestructura. (Jenkins, 2011, citado en Perroti, 2015: 49).

De otro lado, las exportaciones de América Latina compiten con una diversificada fuente de abastecimiento de bienes primarios y minerales hacia China, tales como, Australia, Canadá, Estados Unidos, Nueva Zelandia y otros países vecinos del país asiático. (Rosales y Kuwayama, 2012, citado en Perroti, 2015: 50).

Durante el período de desaceleración económica 2008-2009, se incrementó la participación de China 
como destino de las exportaciones de la región latinoamericana, en detrimento del peso de Estados Unidos que mantenía hasta entonces, como consecuencia de la política económica contracíclica aplicada por el gobierno chino, consistente en un programa de inversiones de 586,000 millones de dólares en infraestructura, con lo cual China consiguió paliar los efectos de la crisis, logrando crecer 9.1\% en 2009, y la rápida recuperación del comercio exterior, luego del impacto inicial. (Sela, 2010 en Perroti, 2015: 51).

Se destaca la importancia estratégica que tiene China para América Latina como destino de las exportaciones, por consiguiente, la desaceleración del crecimiento chino, así como el cambio de modelo, es motivo de seria preocupación por el impacto que tendría en el mediano plazo para la región.

\section{El comercio exterior peruano en el contexto global}

La estructura y composición del comercio exterior peruano tiene un patrón de especialización orientado a la exportación de productos primarios y de bienes de poco valor agregado y la importación creciente de bienes de alta y mediana tecnología, lo cual representa una de las mayores debilidades de la economía nacional. (Tabla $\mathrm{N}^{\circ} 01$ )

\section{Tabla No 01}

Perú: Composición de Exportaciones e Importaciones

\begin{tabular}{|l|c|c|c|c|c|}
\hline Exportaciones reales & 1987 & 1993 & 1998 & 2003 & 2008 \\
\hline $\begin{array}{l}\text { Productos primarios } \\
\text { BI basados en Recursos Natu- } \\
\text { rales }\end{array}$ & $50.63 \%$ & $41.43 \%$ & $33.85 \%$ & $34.65 \%$ & $41.26 \%$ \\
\hline Primarios + Basados en RN & $80.57 \%$ & $81.51 \%$ & $47.94 \%$ & $50.24 \%$ & $39.98 \%$ \\
\hline BI de Baja Tecnología & $16.08 \%$ & $14.39 \%$ & $13.96 \%$ & $11.92 \%$ & $81.24 \%$ \\
\hline BI de Mediana Tecnología & $3.18 \%$ & $2.41 \%$ & $3.51 \%$ & $2.42 \%$ & $3.72 \%$ \\
\hline BI de Alta Tecnología & $0.16 \%$ & $0.20 \%$ & $0.53 \%$ & $0.25 \%$ & $0.36 \%$ \\
\hline Otros & $0.01 \%$ & $0.06 \%$ & $0.21 \%$ & $0.52 \%$ & $0.54 \%$ \\
\hline Total & $100.0 \%$ & $100.0 \%$ & $100.0 \%$ & $100.0 \%$ & $100.0 \%$ \\
\hline
\end{tabular}

\begin{tabular}{|l|c|c|c|c|c|c|}
\hline Importaciones reales & 1978 & 1987 & 1993 & 1998 & 2003 & 2008 \\
\hline Productos primarios & $8.49 \%$ & $15.37 \%$ & $18.87 \%$ & $22.15 \%$ & $19,2018,88 \%$ & $11.96 \%$ \\
\hline $\begin{array}{l}\text { BI basados en Recursos Na- } \\
\text { turales }\end{array}$ & $25.02 \%$ & $25.14 \%$ & $21.50 \%$ & $18.28 \%$ & $18.88 \%$ & $14.82 \%$ \\
\hline Primarios + Basados en RN & $33.51 \%$ & $40.51 \%$ & $40.37 \%$ & $40.42 \%$ & $38.08 \%$ & $26.79 \%$ \\
\hline BI de Baja Tecnología & $4.26 \%$ & $5.52 \%$ & $9.71 \%$ & $10.71 \%$ & $13.97 \%$ & $15.17 \%$ \\
\hline BI de Mediana Tecnología & $48.41 \%$ & $39.75 \%$ & $36.24 \%$ & $35.81 \%$ & $31.60 \%$ & $41.16 \%$ \\
\hline BI de Alta Tecnología & $8.51 \%$ & $13.44 \%$ & $13.12 \%$ & $12.36 \%$ & $15.55 \%$ & $16.23 \%$ \\
\hline Otros & $5.32 \%$ & $0.78 \%$ & $0.56 \%$ & $0.70 \%$ & $0.80 \%$ & $0.65 \%$ \\
\hline \multicolumn{1}{|c|}{ Total } & $100.0 \%$ & $100.0 \%$ & $100.0 \%$ & $100.0 \%$ & $100.0 \%$ & $100.0 \%$ \\
\hline
\end{tabular}

Fuente: Roca, 2012, p. 26

Esta estructura del comercio que caracteriza a la economía peruana como primario - exportadora, aún no ha podido ser revertida; la misma que viene desde el siglo XIX tal como afirma Contreras “... hasta finales del siglo diecinueve tres cuartas de la población vivían inmersas en un régimen autárquico, y cuyo sector comercial consistía en economías primarias de exportación diseminadas por el territorio de la república.” (Contreras, 2009:9)

El proceso de liberalización comercial de la economía peruana se inició en 1990 con una reforma comercial, dándose inicio al proceso de desmantelamiento del sistema proteccionista estructurado durante los últimos gobiernos. En 1991 se profundizó la reforma comercial estableciéndose una estructura arancelaria simplificada de tres niveles (5\%, 15\% y $25 \%$ ), de las cuales el $81 \%$ correspondían a las partidas arancelarias del 15\%. Pasando el arancel promedio del $66 \%$ al $17 \%$ en el lapso de un año, con una disminución en la dispersión de las tasas (Saavedra, 1997:5). Simultáneamente se liberalizaron la cuenta corriente de la balanza de pagos y la de capitales, 
optando el BCR por un sistema cambiario de flotación sucia, con intervenciones permanentes a fin de evitar fluctuaciones severas del tipo de cambio.

Como parte del proceso de liberalización comercial, en 1991 se eliminó el Certificado de Reintegro Tributario (CERTEX), principal instrumento de promoción de exportaciones no tradicionales desde finales de los setenta; siendo reemplazado por el drawback, mecanismo de devolución de impuestos pagados por los exportadores de productos no tradicionales, y que a partir de 1995 equivale a 5\% del valor FOB exportado a todos los productos que en el año anterior registraron ventas al exterior por menos de 10 millones de dólares (Díaz, et al, 2001:9). En 1996, se crea una Comisión para Promoción de Exportaciones del Perú (Prompex) como un organismo estatal autónomo con el objetivo de promover las exportaciones de productos peruanos en el exterior.

Asimismo, se crearon los Centros de Transformación y Comercialización (CETICOS) mediante los cuales, las empresas exportadoras quedan exoneradas de todo impuesto, tasa, aportación o contribución, tanto nacional como municipal, durante los 15 años posteriores al inicio de sus operaciones. Los CETICOS, fueron considerados Zonas Primarias Aduaneras, de tal forma que las mercaderías que ingresen a dichos centras a través de Ilo, Matarani o Paita, eran exentos de pago de derechos arancelarios y demás tributos.

Las exportaciones que prácticamente estaban estancadas hasta 1993, iniciaron un proceso de crecimiento acelerado a partir de 1994, alcanzando en el caso de las exportaciones tradicionales, como el cobre, oro y harina de pesado, una tasa de crecimiento anual de $19.3 \%$, como resultado de un aumento de $80 \%$ en el volumen de oferta exportable durante 1990 y 1997; y en el caso de las exportaciones no tradicionales la tasa promedio fue de $19.1 \%$, sustentada en una mayor diversificación de la oferta exportable. No obstante, el dinamismo generado en las exportaciones se generó en déficit comercial entre $5.0 \%$ y 7.3\%. (BCRP, 1998, citado en Díaz, et al, 2001: 10)

Por su lado, la participación de las importaciones se incrementó en casi 5 puntos porcentua- les del PBI durante los noventa. Derivándose un hecho importante, que la participación de las exportaciones más importaciones en el PBI no llegó al 30\%, a pesar de la apertura comercial y el incremento de las exportaciones. (Díaz, et al, 2001:11)

Durante el período 2005-2014, bajo un contexto externo favorable y una política económica orientada a dar estabilidad de precios en la economía peruana, la tasa de crecimiento promedio del PBI fue de $6.1 \%$ y un nivel de inflación promedio de $2.9 \%$ (BM, 2015). A pesar de la crisis financiera internacional de 2008; después de registrar una contracción de $-12 \%$ en el año 2009, el sector exportador peruano tuvo un sorprendente crecimiento del $33.3 \%$ y $29.4 \%$ en los dos años siguientes 2010 y 2011 (ADEX, 2015a:6), como efecto de la política contracíclica aplicada en la economía china, vinculada a la inversión en infraestructura.

Sin embargo, a partir del año 2012 comienza el declive en las exportaciones, $-0.3 \%$ en $2012,-7.9 \%$ en 2013 y $-9.6 \%$ en 2014, fundamentalmente en sectores industriales como, el textil, confecciones y metalmecánica, los mismos que pierden competitividad frente a los productos del Asia y enfrentan serias restricciones al comercio en la región sudamericana. Además, la crisis de los bonos soberanos europeos, genera una segunda desaceleración de la economía, lo cual evidenció la necesidad de una mayor diversificación de mercados de las exportaciones peruanas, ya que en el año 2012, la tasa de crecimiento de las exportaciones tradicionales cayó en -3.3\% y en 10.0\% las no tradicionales. (ADEX, 2015a:7). En el año 2014 la participación porcentual de las exportaciones en el PBI retrocedió a 19.4\% desde un $27.2 \%$ en 2011 , regresando a la participación porcentual del año 1978. (ADEX, 2015a:8).

La tasa de crecimiento del PBI se desaceleró abruptamente a partir de 2014 alcanzando solo un nivel de $2.4 \%$ en este año, debido principalmente a condiciones externas adversas, asociadas a la caída de los precios de las materias por el cambio de modelo de China, un declive en la confianza interna y una reducción de la inversión, asociados al cambio presidencial. Además de las condiciones climáticas que 
afectaron, la industria pesquera y, la ejecución del programa de inversión pública. Dando como resultado una contracción de $4.8 \%$ en la inversión bruta interna y un nivel de inflación de 3.2\%. (BM, 2015)

En el año 2015, la Asociación de Exportadores del Perú (ADEX) alerta sobre la crisis por la que viene pasando el sector exportador peruano, convocando a las instancias gubernamentales a dar las políticas fiscales y exoneraciones tributarias y laborales sobre las gratificaciones, a fin de retomar el crecimiento. Ya que todos los sectores exportadores estaban en rojo, incluso las agro exportaciones declinaron en $-4.8 \%$ hacia abril de 2015 . El conjunto de las exportaciones no tradicionales, aquellas que representan alrededor del $35 \%$ de la canasta total y donde descansa el futuro de la diversificación productiva del país, tuvo una merma de $7.5 \%$ en el período de enero a junio de 2015. (Adex, 2015a:5-6)

El pobre desempeño de las exportaciones no tradicionales tiene un crucial diagnóstico en el índice de competitividad del World Economic Forum (WEF), donde el Perú pasó de la posición 61 en el 2014 a la 65 en el 2015. El ránking IMD del Centro Mundial de Competitividad registra un descenso para el Perú de 11 posiciones desde el año 2011. Del puesto 43 cayó al 54. Como referencia, Chile se ubica en el puesto 35. (Adex, 2015b:10)

El tema de fondo es la pérdida de competitividad en actividades intensivas en mano de obra frente a productos del Asia; además de enfrentar la contracción del mercado norteamericano y las restricciones al comercio en Ecuador, Venezuela y Argentina. En este año dejaron de exportar 1,938 empresas mientras que en 2014 salieron de la actividad 1,822 empresas, lo cual representa una pérdida de empleos y capacidad exportadora muy difícil de recuperar (ADEX, 2015a:7).

La agenda pendiente según Adex, son los problemas tributarios, laborales y logísticos, para lo cual se plantean reformas institucionales, la reforma tributaria y la reforma laboral. No obstante, se considera que éstas serían medidas de corto plazo, ya que en el mediano plazo se tiene el fundado temor que no habrá retorno al crecimiento anterior, debido a la reestructuración de la economía global cuyo centro de gravedad se traslada a el Asia y debido también al agotamiento del modelo de los TLCs bilaterales, los cuales ya no tienen el mismo impacto anterior porque muchos países firmaron acuerdos con las principales economías del mundo.

\section{CONCLUSIONES}

1. Una de las tendencias de la economía mundial, es el alcance cada vez más global de las crisis macroeconómicas, mostrando hasta qué punto las economías en desarrollo y las economías emergentes dependen del proceso cíclico originado en las economías desarrolladas y de los vínculos comerciales y financieros y la importancia de las cadenas de suministro en la propagación de las crisis. Así, entre 2003 y 2008, los precios de la energía, metales y minerales se duplicaron con creces, como consecuencia de la expansión de varias economías en desarrollo del Grupo G-20 basada en la utilización intensiva de recursos naturales y energéticos. Sin embargo, después de la crisis global de 2008-2009 el comercio mundial ha perdido su dinamismo, evidenciándose a partir del año 2012, con variaciones negativas registradas en el índice de precios de las exportaciones mundiales, en un contexto de menores precios de los productos básicos como el petróleo y los metales.

2. China representa un socio comercial estratégico para América Latina; durante el período de desaceleración económica 2008-2009, se incrementó la participación de este país como destino de las exportaciones de la región latinoamericana, en detrimento del peso que Estados Unidos que mantenía hasta entonces; como consecuencia de la política económica contracíclica aplicada por el gobierno chino. Por consiguiente, la desaceleración del crecimiento chino, así como el cambio de su modelo económico, es motivo de preocupación por el impacto que tendría en el mediano plazo para la región, sobre todo cuando existe un agotamiento del modelo de los TLCs bilaterales, los cuales ya no tienen el mismo impacto anterior porque muchos países firmaron acuerdos con las principales economías del mundo. 
3. El acelerado crecimiento de las exportaciones peruanas principalmente de bienes primarios, se inicia con el proceso de liberalización y estabilización de la economía, a partir de los años noventa, y está ligado al fuerte aumento de precios por la gran demanda china de estos bienes, a partir del año 2000; sin embargo, la crisis internacional de 20082009 y el inicio del cambio de modelo económico chino, incidieron en el proceso de declive de las exportaciones peruanas a partir del año 2012; acentuado por la fuerte caída de los precios de las materias primas, la pérdida de competitividad frente a los productos asiáticos y la estructura y composición del comercio exterior peruano, cuyo patrón de especialización está orientado a la exportación de bienes primarios y bienes de poco valor agregado.

\section{BIBLIOGRAFÍA}

1. Asociación de Exportadores del Perú (ADEX). (2015a). Crisis exportadora. ¿Qué hacemos para reflotar el sector?, Rev. Perú Exporta $\mathrm{N}^{\circ}$ 393, abril/junio, Lima, Perú.

2. Asociación de Exportadores del Perú (ADEX). (2015b). Exportaciones para que piquen, Rev. Perú Exporta N³94, agosto, Lima, Perú.

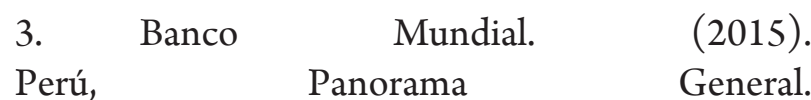

4. BOUBY, FRANCISCA. (1998). El TLC en el contexto de la globalización y regionalización. El caso del Sector Automotriz Terminal Mexicano. Tesis de Maestría, UNMSM, Facultad de Economía, Lima, Perú.

5. CEPAL. (2015). Panorama de la Inserción Internacional de América Latina y el Caribe. La crisis del comercio regional: diagnóstico y perspectivas, Naciones Unidas, Santiago de Chile

6. CONTRERAS CARLOS. (2009). Política demográfica, crecimiento económico y formación del mercado laboral en el Perú del siglo veinte. Departamento de Economía de la PUCP. Rev. Investigaciones de Historia económica invierno, número 13. Pp. 11 a 41

7. Cornejo Enrique (1996). Comercio internacional, hacia una gestión competitiva. Edit. San Marcos, Lima, Perú.

8. KRUGMAN PAUL. (1988). La nueva teoría del comercio internacional y los países menos desarrollados, El Trimestre Económico, №217, ene-mar de 1988, pp. 41-42

9. KRUGMA, PAUL. (2009). El retorno de la economía de la depresión y la crisis actual. Huertas Industrias Gráficas SA., Madrid. 
10. KRUGMAN PAUL. (2012). 14. ROCA, SANTIAGO. (2012). EspecializaDetengamos esta crisis ya. Edito- ción tecnológica en el comercio exterior del Perú: un rial Paidós Mexicana, SA., México DF. análisis comparado con Corea, China, Colombia y México. Revista de Ciencias Sociales (Ve), vol. XVI11. Organización Mundial del Comercio II, núm. 1, enero-marzo, 2012, pp. 24-40. Universi(OMC). (2014). Informe sobre el comercio mun- dad delZulia, Maracaibo, Venezuela. Disponible en: dial 2014. Comercio y Desarrollo tendencias re- 15. http://www.redalyc.org/articulo. cientes y función de la OMC, Ginebra, Suiza oa? $\mathrm{id}=28022785003$

12. PERROTI, D. (2015). La República Popular China y América Latina: impacto del crecimiento económico chino en las exportaciones latinoamericanas, Cepalrevista $\mathrm{N}^{\circ} 116$, agosto, Santiago deChile.

13. PIKETTY, THOMAS. (2014). El capital en el siglo XXI. Edit. Fondo de Cultura Económica, México, D.F.

16. SAAVEDRA, JAIME.(1997). Liberalización comercial e industria manufacturera en el Perú. Lima: CIES. 107 p. Investigaciones breves, 2. 17. STIGLITZ,JOSEPHE.(2012).Elprecio de la desigualdad. Santillana Edic. Generales, España.

\section{REFERENCIAS ELECTRÓNICAS}

18. http://www.bancomundial.org/es/country/peru/overview\#1 (visitado el 30.06.16) 